\title{
Dynamic Assessment in Patients Following Bone-Patellar Tendon-Bone Autograft Anterior Cruciate Ligament Reconstruction ${ }^{\S}$
}

\author{
Roger Lyon ${ }^{*}, 1$ Xue-Cheng Liu ${ }^{1}$, John Hung ${ }^{1}$ and Thomas W. Kernozek ${ }^{2}$ \\ ${ }^{I}$ Department of Orthopaedic Surgery, Children's Hospital of Wisconsin, Medical College of Wisconsin, 9000 W. \\ Wisconsin Ave., P.O. Box 1997, Suite C360, Milwaukee, WI 53201, USA \\ ${ }^{2}$ Department of Health Profession, University of Wisconsin-La Cross, La-Cross, WI 54601, USA
}

\begin{abstract}
Background: The knee's passive movement is insufficient to determine function in patients following ACL reconstruction.

Hypothesis: We hypothesize that there are kinematic differences in the lower extremities (LE) during stair climbing and ground level walking following ACL surgery between the intact and reconstructed sides.

Study Design: This was a retrospective cohort study. Eleven patients with an average age of 15.3 years at the time of their ACL reconstructive surgery (BPTB autograft) participated in the study.

Methods: Patients were followed for at least 2 years post surgery. The subjects underwent a non-weight bearing ability test to reproduce predetermined knee joint positions. Their LE's velocity and joint kinematics were then measured during level ground walking and on a set of custom designed stairs as they ascended and descended.

Results: During level ground walking the maximum internal rotation at the ankle during the swing phase on the reconstructed side increased significantly from $2.3^{\circ}$ to $19.9^{\circ}$ compared to the unreconstructed $\operatorname{limb}(\mathrm{P}=0.04)$. The leading reconstructed knee during stair ascent exhibited less knee flexion as compared to the unreconstructed knee for each step $\left(1^{\text {st }}\right.$ step: $74.2^{\circ} v s 81.5^{\circ} ; 2^{\text {nd }}$ step: $93.6^{\circ}$ vs $97.6^{\circ} ; 3^{\text {rd }}$ step: $48^{\circ}$ vs $53.5^{\circ} ; 4^{\text {th }}$ step: $72.5^{\circ}$ vs $\left.78.1^{\mathrm{o}} ; \mathrm{p}<0.05\right)$.

Conclusions: A two-year follow-up study in adolescents who had a bone-patellar tendon-bone autograft demonstrated that they had normal knee proprioception and 3D joint rotations of the LE, while showing an alteration of the ankle and knee kinematics during walking or ascending stairs.
\end{abstract}

Keywords: ACL, gait analysis, proprioception, stair.

\section{INTRODUCTION}

The anterior crucial ligament (ACL) helps constrain anterior displacement of the tibia on the femur and plays a role in varus/valgus knee stability [1]. As many as $65 \%$ of acute knee hemarthrosis in children have an ACL injuries [2]. Injuries to this ligament have been shown to lead to degenerative changes in the knee, especially in the pediatric population [3]. Functionally, these injuries often present symptoms of "giving away". Reconstruction in the young adult is highly recommended, due to the high potential for progressive wear [4-6]. A number of techniques for ACL reconstruction have been proposed and examined. These include allograft, autograft and extra-articular reconstruction. Most surgeons prefer autografts with either the bone- patellar tendon-bone (BPTB) or the semitendinosus/gracilis tendons $(\mathrm{ST} / \mathrm{G})[7]$.

\footnotetext{
*Address correspondence to this author at the Department of Orthopaedic Surgery, Children's Hospital of Wisconsin, 9000 W. Wisconsin Ave., PO. Box 1997, Suite C360, Milwaukee, WI 53201, USA; Tel: 414.337.7325; Fax: 414.337.7337; E-mail: rlyon@chw.org
}

${ }^{\S}$ Study conducted at Children's Hospital of Wisconsin, Medical College of Wisconsin, Milwaukee, Wisconsin, USA
There is a significant risk of recurrent knee injury as a result of an ACL injury. There are several studies that demonstrate impaired proprioception of the knee [8-10]. Proprioception is referred to as the conscious perception of limb position in space and is facilitated by receptors (Ruffini endings, Pacinian corpuscles, Golgi tendon organs and free nerve endings) [8-10]. These receptors are found in the skin, ligaments, capsule, tendons and menisci of the knee. The majority of the joint mechanoreceptors are located at the tibial and femoral insertions of the ligaments, most of which are subsynovial [8-10]. Reproducing the passive range of motion to measure knee proprioception does not give as accurate results as the quantitative methods used in a motion analysis. Examining normal functional activities, i.e. stair climbing, may be more useful than measuring the knee's passive range of motion for the assessment of dynamic proprioception.

There are multiple studies in the adult population following ACL injuries and reconstruction [9-11]. The studies evaluated functional impairments using gait analyses and found reductions of peak knee moment and power after ACL reconstruction. The studies also showed that other joints besides the knee were affected, e.g. the ankle had significantly increased ankle excursion and moment contralaterally [9]. Significant reductions in joint flexion were found at the hip, knee and ankle during the stance phase six months after surgery compared with 3 weeks 
after surgery [11]. Neuromuscular adaptation can significantly influence dynamic function in subjects with ACL reconstruction (either BPTB or ST/G procedure) during activities such as walking, jumping, ascending the stairs or descending the stairs [8-10]. However, none of these studies have investigated gait adaptation in individuals near skeletal maturity following BРТВ during stair ascending and descending. It is important to have an understanding of joint kinematics and gait adaptation for younger subjects who have undergone ACL reconstruction.

The purpose of this study is threefold: 1) To compare the reproducibility of knee flexion angles at $30^{\circ}$ and $60^{\circ}$ in patients who have had ACL reconstruction with patellar bone-tendonbone autografts on both the unaffected and affected sides; 2) To evaluate kinematic differences of the knee in patients status post ACL reconstruction when ascending and descending a short staircase with varying step heights; 3) To compare the kinematics of affected and unaffected lower extremities during level ground walking.

\section{METHODS}

The average age of the patients at the time of ACL reconstruction was 15.3 years. The time from diagnosis of the knee injury to operation averaged 3.8 months. The average age during the gait analysis was 17.8 years, with a range of 14-25 years. Patients were followed for at least 2 years post surgery. Eleven patients were studied, 8 females and 3 males. Four subjects had surgical repair on the left knee, 6 on the right, and 1 had bilateral reconstruction. All of the patients were diagnosed with ACL disruption. MRI results and arthroscopy indicated all patients had complete ACL tears. All patients had a positive Lachman's test. All of the patients received a BPTB autograft ACL reconstruction procedure. The autograft was harvested from the central one third of the patella tendon and used for the arthroscopic ACL reconstruction. The tibial bone tunnel was located using the anatomical foot print of the native ACL and by drilling through the tibial metaphysis $1.5 \mathrm{~cm}$ medial to the tibial tubercle utilizing a standard ACL tibial guide, which was set at 55 degrees. The femoral tunnel was drilled using the transtunnel technique at either a 10:30 or 1:30 position in the femoral notch just anterior to the posterior femoral cortex. Drill Holes were used in the femur and tibia and the autograft was secured using metal interference screws [7]. The autograft was tensioned with the knee at 20 degrees flexion and by posterior drawer force. An anterior draw test and pivot shift test were utilized to check knee stability. Our patients had either physis closure or were within one year of physis closure. Post-operative rehabilitation included immediate full weight-bearing and physical therapy. Sport activities were restricted for 6 months. Post-surgical status: three of the subjects participated in competitive soccer, softball, and/or tennis. The others returned to normal recreational sports. There were no leg length discrepancies more than $1.0 \mathrm{~cm}$ and no report of bony deformities during the follow-up. All patients were operated by the Children's Hospital of Wisconsin's senior orthopedic surgeon (RL) and tested at the Musculoskeletal Functional Assessment Center at the Children's Hospital of Wisconsin. The informed consent of all subjects was obtained prior to testing. The study and consent forms were approved by the IRB committee at the Children's Hospital of Wisconsin. All subjects participated in a non-weight-bearing proprioception assessment (repositioning test), motion analysis during walking, and motion analysis while ascending and descending stairs.
An Electromagnetic Tracking System (ETS) was used to gather subject data. Seven sensors from the ETS were attached to the skin of the L5 region, bilateral thigh, bilateral tibia, and dorsal aspect of the foot to create a seven-segment rigid link model. The Electromagnetic Tracking System used a Star Track transmitter (Polhemus Inc., Colchester, VT), a PC interface and Skill Technologies' 3D motion capture and analysis software (Skill Technologies, Inc., Phoenix, AZ). The sensors were hard wired to a data processing station with cabling. The motion sampling rate was $120 \mathrm{~Hz}$. The ETS has a reported angle error in the three dimensions less than 1 degree and a displacement error of less than $2 \mathrm{~cm}$. The accuracy of the rotation measurements on a static object was $97.5 \%$ [12]. Anatomical landmarks of the lower extremity were digitized with a stylus, which resulted in defining the local coordinates for threedimensional measurements. Subjects were allowed to ambulate in a straight line without constraints. The three-dimensional joint rotations, including the flexion/ extension in the sagittal plane, inversion/eversion in the coronal plane, and internal /external rotation in the transverse plane of each lower extremity and pelvis were calculated. In the stance phase and swing phase, maximal and minimal values of the 3D kinematics were identified. Also temporal and distance data were evaluated. In all subjects (excluding one), the contralateral non-reconstructed limb was used as the control.

During the non-weight-bearing proprioception repositioning test [13], the subjects were seated with a standardized starting position of $90^{\circ}$ of hip flexion and knee extension. At first, the investigators moved their knees from the starting position to either $30^{\circ}$ or $60^{\circ}$ flexion, then subjects actively moved their knees while wearing sleep goggles (eyes closed), as instructed by the investigator, to two flexion angles $\left(30^{\circ}\right.$ and $\left.60^{\circ}\right)$ in a randomized fashion, as measured by the ETS. A total of three trials for each knee at each angle were conducted.

Next the patients walked along a 15-meter-walkway at a self-selected speed. Three trials for each leg were recorded with the ETS.

Lastly, a customized four-step staircase was mounted in the middle of the walkway. This staircase was designed to enable a more dynamic measurement of the knee joint. Each step had a different height, $18 \mathrm{~cm}, 28 \mathrm{~cm}, 8 \mathrm{~cm}$, and $18 \mathrm{~cm}$, respectively. All steps had a depth of $28 \mathrm{~cm}$, and a width of $120 \mathrm{~cm}$. Patients were instructed to ascend and descend the stairs at a natural speed with their eyes opened for three practice trials. There were no handrails on the staircase. Each patient was spotted by one of the investigators while ascending and descending the staircase blindfolded. The patients performed this task with their reconstructed leg leading and their uninvolved leg trailing on one trial, then the uninvolved leg leading and reconstructed leg trailing on the next trial. Three trials for each leg were recorded.

The Wilcoxon matched pair test was applied to compare the affected and unaffected side in the assessment of reproducing knee angle, kinematics of level walking and steps as decent and ascent, because of the non normal distribution of kinematic variables. A p-value of less than 0.05 was considered significant.

\section{RESULTS}

In total, 31 kinematic parameters were evaluated, including walking velocity, maximum or minimum flexion/extension, inversion/eversion, and internal/external rotation during the 
stance phase or swing phase at the pelvis, hip, knee, and ankle joint. There were no statistically significant differences between the reconstructed limbs compared with the contralateral limb in the reproduction of non-weight bearing knee joint angles $(\mathrm{P}>0.05)$. The differences in knee flexion angles between the reconstructed and non-reconstructed knees while bending the knee at $30^{\circ}$ and $60^{\circ}$ were approximately $2^{\circ}$ and $1^{\circ}$.

There was no significant difference in level walking velocity between the reconstructed limb $(1.14 \mathrm{~m} / \mathrm{s})$ and the contralateral side $(1.19 \mathrm{~m} / \mathrm{s})(\mathrm{P}>0.05)$. There was no statistical difference in the hip and knee kinematics between the reconstructed and contralateral sides during level ground walking $(\mathrm{P}>0.05)$, except for a maximal pelvic elevation and declination, pelvic protraction, and ankle internal rotation $(\mathrm{p}<0.05)$ (see Table 1). Although there were statistical differences of the pelvic obliquity and protraction, the range of motion (ROM) of the pelvis in the coronal plane between the two limbs remained close. The ranges of motion for the reconstructed limb and normal limb are: $37.8^{\circ}$ vs $37.9^{\circ}$ at the hip; $69.4^{\circ}$ vs $73.8^{\circ}$ at the knee; and $32.9^{\circ}$ vs $34.7^{\circ}$ at the ankle $(\mathrm{P}>0.05)$. The ankles maximum internal rotation during the swing phase on the affected side significantly increased $\left(19.9^{\circ}\right)$ compared to the uninvolved side $\left(2.3^{\circ}\right)(\mathrm{p}=0.04)$.

Overall the unreconstructed contralateral knee exhibited increased angular velocities compared with those of the operated knee at each step during ascent or decent. Except for reduced angular velocity $(3.82 \mathrm{deg} / \mathrm{s})$ on the leading reconstructed knee during ascent for the $4^{\text {th }}$ step as compared to the leading contralateral knee $(4.30 \mathrm{deg} / \mathrm{s})(\mathrm{p}=0.02)$, there was no difference in angular velocity in ascending and descending stairs of affected and unaffected knees $(p<0.05)$. There were four curves during ascent or descent, where each curve consisted of two peaks and two valleys (Fig. 1). There were no differences in the knee kinematics while ascending the stairs between the reconstructed and uninvolved trailing leg, as well as either leading or trailing leg during step descent. However, the leading reconstructed knee during stair climbing exhibited less knee flexion as compared to leading uninvolved knee for each step ( $1^{\text {st }}$ step: $74.2^{\circ}$ vs $81.5^{\circ} ; 2^{\text {nd }}$ step: $93.6^{\circ}$ vs $97.6^{\circ} ; 3^{\text {rd }}$ step: $48^{\circ}$ vs $53.5^{\circ} ; 4^{\text {th }}$ step: $72.5^{\circ}$ vs $\left.78.1^{\circ} ; \mathrm{p}<0.05\right)$ (Table 1). Overall the ROMs on the leading reconstructed limb during stair climbing are less than those on the uninvolved limb from step 1 to step 3 . The leading reconstructed limb during stair climbing showed a significantly reduced ROM at the knee $\left(104^{\circ}\right)$ compared to the unaffected limb $\left(109.6^{\circ}\right)$ for step 4 $(\mathrm{P}=0.04)$.

\section{DISCUSSION}

The present study examined the clinical and functional outcomes of patients following patellar bone-tendon-bone autograft reconstruction at a two years follow-up. The results yielded three important findings. First, patients retained the ability to reproduce knee angles without any significant difference compared to their contralateral control limbs. Secondly, during level walking, their ankles showed a remarkable increased internal rotation on the affected side during the swing phase. Third, they were able to safely ascend and descend a staircase with steps of varying heights without difficulty, but the reconstructed knee demonstrated reduced flexion when it lead during stair climbing.

During ascent of the staircase there is a swing phase and stance phase (see Fig. 1). In the swing phase (from V1 to P1 and P1 to V2), the leading knee is typically flexed during the swing phase to prepare for foot contact. The stance phase can be divided into two periods, one is an initial stance phase (from V2 to P2) during early weight bearing (a single leg support); a second (from P2 to next V1) is in full stance phase with weight bearing (double leg support). The ETS has been routinely used in our Motion Analysis Laboratory. In a previous study, we compared the reproducibility of the ETS measurements with other authors [12]. Intraclass coefficient (ICC) ranged from 0.62 to 0.94 . This indicates the ETS is reliable in the assessment of the lower extremity for younger subjects with the BРTВ reconstruction [14].

The proprioceptive capability of the knee following an ACL injury has been evaluated. It has been demonstrated that proprioception does become significantly compromised with ACL-deficiency [15]. Pap et al. demonstrated a proprioceptive

Table1. Significant Differences Between ACL Reconstruction and Contralateral Knee During Walking or Stair Climbing (Mean \pm SD, Degrees)

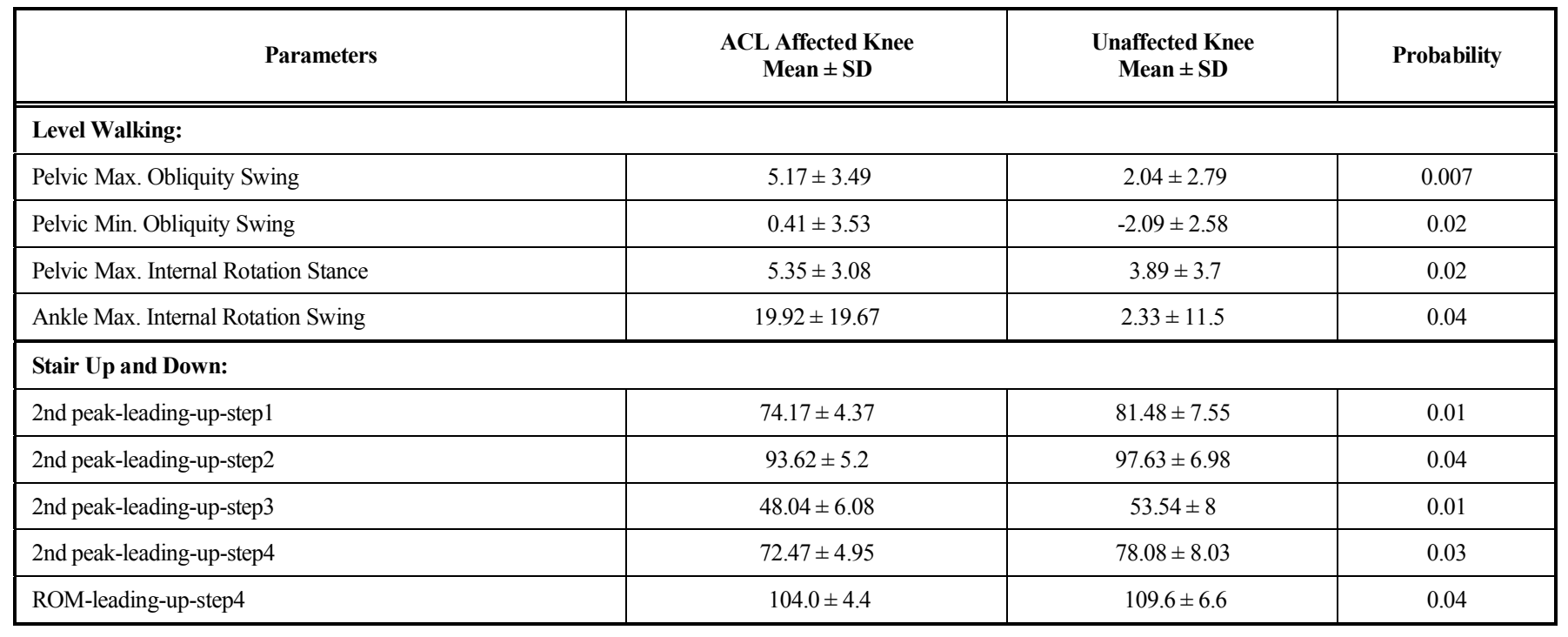


deficit in the absence of the ACL. They tested 20 patients and 15 control subjects in detection of passive joint movement to measure proprioception in the knee. They showed that there were no differences in threshold levels of detection of movement [15]. However, there were differences in the ability to detect the starting and stopping of movement. These differences decreased with increasing angular velocity. In our study, knee movement at time of P2 showed significant differences at the initiation of knee extension.

\section{Knee Flexion/Extension}

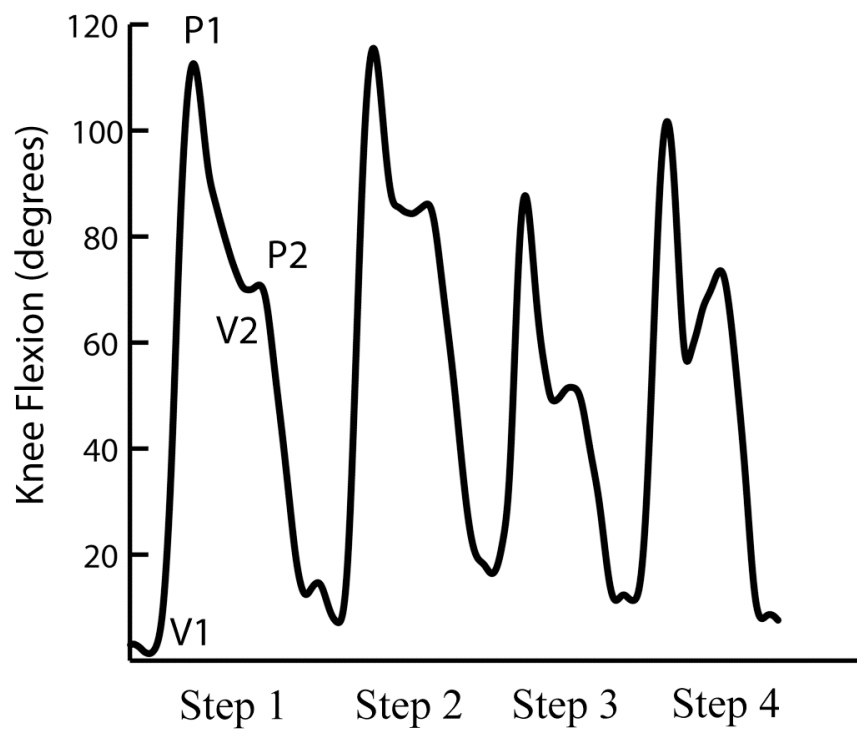

Fig. (1). Flexion and extension of the leading knee joint during ascent in the sagittal plane. Each curve represents a step (in the upward direction), where the first curve represents a normal step (step1), the second a high step (step 2), the third a low step (step 3) and finally a normal step (step 4): (a) knee flexion during a double limb support before ascending as V1; (b) maximum knee flexion in the air before the foot placing on the step, which is represented by $\mathrm{P} 1$; (c) knee extension when the foot rests on the step but non weight bearing (just before a single limb support) as V2; (d) knee flexion with weight bearing at just initial stage of the body weight transfer as $\mathrm{P} 2$.

Fischer and Jensen et al. studied the performance and proprioception differences between groups of non-reconstructed ACL-deficient patients with another group of ACL-reconstructed patients [6, 13, 16-19]. Proprioceptive tests demonstrated a decreased ability to recognize and reproduce a prior angle from a start position of $60^{\circ}$ of flexion. However, we followed up patients with reconstructed knees at an average of 2.5 years after surgery and they showed no differences during passive joint position testing. These results have been shown to restore nonweight bearing proprioception in knees with patellar tendon bone autografts following ACL ruptures. Others agreed that ACL reconstruction did improve or restore proprioceptive deficits following ACL injuries $[4,5]$.

The recovery of the proprioception and function is not attributed to simply reconstructing the ACL. Several studies have demonstrated that proper rehabilitation as well as the recovery time of the ACL reconstruction is important in the recovery of functional mobility in the ACL-reconstructed knee [20]. Lutz et al. reported that isometric closed-kinetic-chain exercises produced significantly less tibiofemoral shear force with comparable muscular activity when compared with isometric open-kinetic-chain exercises [21]. These results provide the rationale for closed rather than open-kinetic-chain exercises as a means of rehabilitation after injury or following reconstruction of the ACL. The use of bone-patellar tendonbone autograft is standard practice in ACL-reconstruction. Our findings suggest that patients that undergo ACL reconstruction with bone-patellar tendon-bone autograft have good but not complete restoration of proprioception. They retain the ability to perform static and dynamic activities without significant deficits.

There is some evidence suggesting that there may be proprioceptive deficits due to the harvesting of the patellar tendon autograft. Kowalk et al. assessed subjects prereconstruction and post-reconstruction in terms of function following bone-tendon-bone autograft reconstruction [9]. They stated that the restoration of the anterior-posterior knee stability was achieved. However, in their 6-month short-term follow-up the injured knee demonstrated reductions in peak moment, power and work. They attributed this to the harvesting of the ipsilateral patellar bone-tendon-bone autograft, and the morbidity associated with the graft. In contrast, our results showed that there was good functional recovery during walking and stair climbing. Our two-year follow-up may have allowed these changes to diminish or reverse. Reider et al. reported that when ACL reconstruction was achieved with either BPTB or quadrupled hamstring/gracilis autograft, there were no significant differences of joint position sense and threshold to detection of passive motion between the patients with BPTB versus hamstring /gracilis grafts [19]. Ristanis et al. found that a two-year follow up in patients with BPTB showed an increase in tibial internal rotation, which is similar to what was evaluated in the patients with ST/G $[22,23]$. The increased internal rotation of the ankle joint in the ACL reconstructed limb from our study may be explained with either a lack of posterolateral bundle reconstruction or the femoral tunnel positioning around the 11-o'clock position, which is unable to provide an appropriate tension or lever arm for the rotational torque [22, 23].

Rudroff examined functional tests, via two legged-jumps, one legged-jumps, squatting and walking [7]. They examined three groups in the 40 soccer players: with BPTB, semitendinosus, and control. Their results demonstrated that the BPTB reconstruction patients had less knee flexion during jumps, compared to the semitendinosus group and control group $\left(36.71^{\circ} \pm 4.26^{\circ}, 45.23^{\circ} \pm 3.72^{\circ}, 59.08^{\circ} \pm 4.04^{\circ}\right.$, respectively). This may indicate increased potential for non-contact ACL injuries due to lack of knee flexion angles with jumping. Additionally, the BPTB group also exhibited lower coactivation of the quadriceps and hamstring muscles. This may suggest better prevention of injuries in the semitendinosus group, due to altered co-activation of the quadriceps and hamstring muscles. However, we have not detected knee movement changes during level walking, only excessive ankle internal rotation. Additionally, there is decreased knee flexion during stair climbing. This may be caused by the reduced knee extensor moment. The reduced knee extensor moment phenomena was reported in patients with ACL deficiency and referred as the quadriceps avoidance pattern [24, 25]. Several studies found that at 6 to 8 months after ACL reconstruction patients demonstrated knee extensor moments close to those of a control group or demonstrated a mild reduction of the knee 
extensor moment $[11,26]$, but another study showed ACLreconstructed patients walked with significant reduction of the knee extensor moment even after 10 months surgery [27]. Although our 2-year follow-up still demonstrated the existence of a quadriceps avoidance pattern during stair ascending, we thought that it may be a compensatory mechanism to balance the agonistic and antagonistic muscles around the knee and to further stabilize the reconstructed knee following the BPTB procedure.

This study demonstrates that the patients who underwent ACL reconstruction in adolescents using bone tendon bone autograft show near normal knee proprioception when assessed by the standard joint angle reproduction test. Functional assessment with level walking and stair climbing show normal limb mechanics, although there were altered kinematics with an increase in ankle internal rotation during the swing phase of level walking and knee extension during stair climbing on the operated limb. Further study is needed to explore the effects of different positions of BPTB on the rotation tibia.

\section{REFERENCES}

[1] Markolf KL, Burchfield DM, Shapiro MM, Shepard MF, Finerman G, Slauterbeck JL. Combined Knee Loading States that Generate High Anterior Cruciate Ligament Forces. J Ortho Res 1995; 13: 930-35.

[2] Kocher M, Saxon HS, Hovis WD, Hawkins RJ. Management and complications of anterior cruciate ligament injuries in skeletally immature patients: surgery of the herodicus society and the ACL study group. J Pediatr Orthop 2002; 22: 452-6.

[3] Graf BK, Lange RH, Fujisaki CK, Landry GL, Saluja RK. Anterior cruciate ligament tears in skeletally immature patients meniscal pathology at presentation and after attempted conservative treatment. Arthroscopy 1992; 8(2): 229-33.

[4] Ahmad CS, Stein BE, Jeshuran W, Nercessian OA, Henry JH. Anterior cruciate ligament function after tibial eminence fracture in skeletally mature patients. Am J Sports Med 2001; 29(3): 339-45.

[5] Iwasa J, Ochi M, Adachi N, Tobita M, Katsube K, Uchio Y. Proprioceptive improvement in knees with anterior cruciate ligament reconstruction. Clin Orthop Relat Res 2000; 381: 168-76.

[6] Roberts D, Andersson G, Fridén T. Knee joint proprioception in ACLdeficient knees is related to cartilage injury, laxity and age: a retrospective study of 54 patients. Acta Orthop Scand 2004; 75(1): 7883.

[7] Rudroff T. Functional capability is enhanced with semitendinosus than patellar tendon ACL repair. Med Sci Sport Exerc 2003; 35(9): 1486-92.

[8] Fremerey RW, Lobenhoffer P, Zeichen J, Skutek M, Bosch U, Tscherne H. Proprioception after rehabilitation and reconstruction in knees with deficiency of the anterior cruciate ligament. J Bone Joint Surg (Br) 2000; 82B(6): 801-6.

[9] Kowalk DL, Duncan JA, Mccue FC, Vaughan C. Anterior cruciate ligament reconstruction and joint dynamics during stair climbing. Med Sci Sport Exerc 1997; 29(11): 1406-13.
[10] Lephart SM, Pincivero DM, Giraldo JL, Fu FH. The role of proprioception in the management and rehabilitation of athletic injuries. Am J Sports Med 1997; 25(1): 130-7.

[11] Devita P, Hortobagyi T, Barrier J. Gait biomechanics are not normal after anterior cruciate ligament reconstruction and accelerated rehabilitation. Med Sci Sports Exerc 1998; 30: 1481-8.

[12] Kobayashi K, Gransberg L, Knutsson E, Nolen P. A new system for three-dimensional gait recording using electromagnetic tracking. Gait and Posture 1997; 6: 63-75.

[13] Fischer-Rasmussen T, Jensen PE. Proprioceptive sensitivity and performance in anterior cruciate ligament-deficient knee joints. Scand J Med Sci Sport 2000; 10(2): 85-9.

[14] Liu XC, Thometz J, Lyon R, Broudeau C. Six-segmental foot model using ETS. 20 ${ }^{\text {th }} \mathrm{ISB} / 29^{\text {th }}$ ASB, Cleveland, OH, 07/31-08/05, 2005.

[15] Pap G, Machner A, Nebelung W, Awiszus F. Detailed analysis of proprioception in normal and ACL-deficient knees. J Bone Joint Surg (Br) 1999; 81B(5): 764-8.

[16] Fischer-Rasmussen T, Jensen TO, Kjaer M, Krogsgaard M, DyhrePoulsen P, Magnusson SP. Is proprioception altered during loaded knee extension shortly after ACL rupture? Int J Sports Med 2001; 22(5): 385-91.

[17] Hopper DM, Creagh MJ, Formby PA, Goh SC, Boyle JJ, Strauss GR. Functional measurement of knee joint position sense after anterior cruciate ligament reconstruction. Arch Phys Med Rehabil 2003; 84(6): 868-72.

[18] Jensen TO, Fischer-Rasmussen $T$, Kjaer M, Magnusson SP Proprioception in poor- and well-functioning anterior cruciate ligament deficient patients. J Rehabil Med 2002; 34(3): 141-9.

[19] Reider B, Arcand MA, Diehl LH, et al. Proprioception of the knee before and after anterior cruciate ligament reconstruction. Arthroscopy 2003; 19(1): 2-12.

[20] Liu-Ambrose T, Taunton JE, MacIntyre D, McConkey P, Khan KM. The effects of proprioceptive or strength training on the neuromuscular function of the ACL reconstructed knee: a randomized clinical trial. Scand J Med Sci Sports 2003; 13(2): 115-23.

[21] Lutz GE, Palmitier RA, Chao EYS. Comparison of tibiofemoral joint forces during open-kinetic chain and closed kinetic chain exercises. J Bone Joint Surg (Am) 1993; 75(5): 732-8.

[22] Georgoulis AD, Ristanis S, Chouliaras V, Moraiti C, Stergiou N. Tibial rotation is not restored after ACL reconstruction with a hamstring graft. Clin Orthop Relat Res 2006; 454: 89-94.

[23] Ristanis S, Stergiou N, Patras WK, Tsepis E, Moraiti C, Georgoulis AD. Follow-up evaluation 2 years after ACL reconstruction with bonepatellar tendon-bone graft shows that excessive tibial rotation persists. Clin J Sport Med 2006; 16: 111-6.

[24] Patel RR, Hurwitz DE, Andriacchi TP, et al. Mechanisms for the 'quadriceps avoidance gait' seen in ACL deficient individuals. Gait Posture 1997; 5 (2): 147.

[25] Wexler G, Hurwitz DE, Bush-Joseph CA, et al. Functional gait adaptations in individuals with anterior cruciate ligament deficiency over time. Clin Orthop Relat Res 1998; (348): 166-175.

[26] Bush-Joseph CA, Hurwitz DE, Patel RR, et al. Dynamic function after anterior cruciate ligament reconstruction with autologous patellar tendon. Am J Sports Med 2001; 29 (1): 36-41.

[27] Timoney JM, Inman WS, Quesada PM, et al. Return of normal gait patterns after anterior cruciate ligament reconstruction. Am J Sports Med 1993; 21 (6): 887-9. 